\title{
Left frontal glioma induces functional connectivity changes in syntax-related networks
}

\author{
Ryuta Kinno 1,2,3*, Shinri Ohta ${ }^{1,3}$, Yoshihiro Muragaki ${ }^{3,4}$, Takashi Maruyama ${ }^{3,4}$ and Kuniyoshi L Sakai ${ }^{1,3}$
}

\begin{abstract}
Background: A glioma leads to a global loss of functional connectivity among multiple regions. However, the relationships between performance/activation changes and functional connectivity remain unclear. Our previous studies (Brain 137:1193-1212; Brain Lang 110:71-80) have shown that a glioma in the left lateral premotor cortex or the opercular/triangular parts of the left inferior frontal gyrus causes agrammatic comprehension accompanied by abnormal activations in 14 syntax-related regions. We have also confirmed that a glioma in the other left frontal regions does not affect task performances and activation patterns.

Results: By a partial correlation method for the time-series functional magnetic resonance imaging data, we analyzed the functional connectivity in 21 patients with a left frontal glioma. We observed that almost all of the functional connectivity exhibited chaotic changes in the agrammatic patients. In contrast, some functional connectivity was preserved in an orderly manner in the patients who showed normal performances and activation patterns. More specifically, these latter patients showed normal connectivity between the left fronto-parietal regions, as well as normal connectivity between the left triangular and orbital parts of the left inferior frontal gyrus.
\end{abstract}

Conclusions: Our results indicate that these pathways are most crucial among the syntax-related networks. Both data from the activation patterns and functional connectivity, which are different in temporal domains, should thus be combined to assess any behavioral deficits associated with brain abnormalities.

Keywords: Agrammatic comprehension, Frontal regions, Functional connectivity, Functional MRI, Glioma, Neural network

\section{Findings \\ Background}

Gliomas are generally considered to cause hyperexcitability throughout the entire brain, leading to epileptic seizures (de Groot et al. 2012). A magnetoencephalogram study of the resting-state functional connectivity showed that a glioma disrupted functional connectivity across not only the regions it occupied, but also other distant regions in both hemispheres of the cerebral cortex (Bartolomei et al. 2006). A recent functional magnetic resonance imaging (fMRI) study with a verb-generation task reported that a single glioma partially reduced the functional connectivity among the bilateral frontal and

\footnotetext{
*Correspondence: kinno@med.showa-u.ac.jp

1 Department of Basic Science, Graduate School of Arts and Sciences,

The University of Tokyo, 3-8-1 Komaba, Meguro-ku, Tokyo 153-8902, Japan

Full list of author information is available at the end of the article
}

temporal regions (Briganti et al. 2012). In such lesion studies, however, not only functional connectivity but activation patterns should be clarified, because their relationships may depend on any tasks and neural networks involved.

We have previously shown that a glioma in the left lateral premotor cortex (LPMC) or in the opercular/triangular parts of the left inferior frontal gyrus (F3op/F3t) is sufficient to cause agrammatic comprehension (Kinno et al. 2009). Moreover, those agrammatic patients showed abnormal task-related activations in 14 regions (Table 1 ). The behavioral data and activation maps obtained from the normal controls (Normal group) and patients have been reported in our previous paper (Kinno et al. 2014). More specifically, patients with a glioma in the left LPMC (LPMC group) had a more profound deficit in the comprehension of scrambled sentences than that of active

\section{Springer}

(c) 2015 Kinno et al. This article is distributed under the terms of the Creative Commons Attribution 4.0 International License (http://creativecommons.org/licenses/by/4.0/), which permits unrestricted use, distribution, and reproduction in any medium, provided you give appropriate credit to the original author(s) and the source, provide a link to the Creative Commons license, and indicate if changes were made. 
Table 1 Brain regions within three syntax-related networks

\begin{tabular}{|c|c|c|c|c|c|}
\hline Brain region & BA & Side & $x$ & $y$ & $z$ \\
\hline \multicolumn{6}{|l|}{ Network I } \\
\hline F3op/F3t & $44 / 45$ & $L$ & -45 & 18 & 27 \\
\hline IPS & $7 / 39 / 40$ & $\mathrm{~L}$ & -21 & -72 & 51 \\
\hline LPMC & $6 / 8$ & $\mathrm{R}$ & 30 & 3 & 45 \\
\hline F3op/F3t & $44 / 45$ & $\mathrm{R}$ & 33 & 18 & 24 \\
\hline pre-SMA & $6 / 8$ & $M$ & 9 & 24 & 51 \\
\hline pSTG/MTG & $22 / 21$ & $\mathrm{R}$ & 60 & -57 & 3 \\
\hline \multicolumn{6}{|l|}{ Network II } \\
\hline LPMC & $6 / 8$ & $\mathrm{~L}$ & -48 & 3 & 42 \\
\hline $\mathrm{AG}$ & 39 & $\mathrm{~L}$ & -33 & -60 & 18 \\
\hline LG & 18 & $M$ & -3 & -69 & 6 \\
\hline Cerebellar nuclei & & $M$ & -3 & -51 & -27 \\
\hline \multicolumn{6}{|l|}{ Network III } \\
\hline F3t & 45 & $\mathrm{~L}$ & -48 & 33 & 6 \\
\hline $\mathrm{F} 3 \mathrm{O}$ & 47 & $L$ & -36 & 15 & -6 \\
\hline pSTG/MTG & $22 / 21$ & $L$ & -57 & -48 & 0 \\
\hline pMTG/ITG & $37 / 19$ & $L$ & -45 & -69 & 0 \\
\hline
\end{tabular}

Stereotactic coordinates $(x, y, z)$ in the Montreal Neurological Institute space are shown for 14 syntax-related regions, functionally determined in our previous study (Kinno et al. 2014). All but two regions were within the gray matter; the right LPMC and left AG were on the border of the gray matter.

$A G$ angular gyrus, BA Brodmann's area, $F 3 O$ orbital part of the inferior frontal gyrus, F3op/F3t opercular/triangular parts of the inferior frontal gyrus, $F 3 t$ triangular part of the inferior frontal gyrus, IPS intraparietal sulcus, $L$ left, $L G$ lingual gyrus, LPMC lateral premotor cortex, $M$ medial, $p M T G / I T G$ posterior middle/inferior temporal gyri, pre-SMA pre-supplementary motor area, $p S T G$ / MTG posterior superior/middle temporal gyri, $R$ right.

and passive sentences, while patients with a glioma in the left F3op/F3t (F3 group) had a more profound deficit in the comprehension of passive and scrambled sentences than that of active sentences. Furthermore, the LPMC and F3 groups showed abnormal overactivity and/ or underactivity in the 14 regions, while the patients with a glioma in the other left frontal regions (Other group) showed normal performances and activation patterns. These 14 regions were grouped into three syntax-related networks: Network I consists of the left F3op/F3t, left intraparietal sulcus (IPS), right frontal regions, presupplementary motor area, and right temporal regions. Network II consists of the left LPMC, left angular gyrus (AG), lingual gyrus, and cerebellar nuclei. Network III consists of the left ventral frontal and posterior temporal regions. Some functional connectivity may be preserved in the Other group, and we infer that the identification of such preserved connectivity would reveal the most crucial pathways among the syntax-related networks, which has not been possible by the study of normal controls alone. The present study thus underscores the importance of examining functional connectivity changes in brain-damaged patients.

\section{Methods}

Here, we provided an overview of the methods; full details are provided in an additional file (see Additional file 1). We analyzed the functional connectivity in 21 patients (Table 2) and 7 normal participants reported previously (Kinno et al. 2014). The fMRI data were analyzed in a standard manner using statistical parametric mapping (SPM8) software with the "unified segmentation" algorithm, which is a generative model that combines tissue segmentation (excluding "other" tissues like a lesion, etc.), bias correction, and spatial normalization (Ashburner and Friston 2005). All normalized functional images were then smoothed by using an isotropic Gaussian kernel of $9 \mathrm{~mm}$ full-width at half maximum. Low-frequency noise was removed by high-pass filtering at $1 / 128 \mathrm{~Hz}$. For creating an SPM design of each participant, hemodynamic responses induced by task trials were modeled with a boxcar function for $6 \mathrm{~s}$ from the onset of each stimulus, and the boxcar function was convolved with a hemodynamic response function. The functional connectivity among multiple regions was assessed by a partial correlation method for the time-series fMRI data (Smith 2012). Using the MarsBaR-toolbox (http://marsbar.sourceforge.net/) for the SPM design, the time-series data were first averaged within a sphere of $6-\mathrm{mm}$ radius centered at the local maximum of each region (Table 1 ). To discount the global differences of signal changes among the runs, the averaged time-series from each of all tested runs were normalized to those of the first run. Using the concatenated runs of each participant, we calculated partial correlation coefficients for each of the time-series of two regions in question; we regressed out all the other nodes, before estimating the correlation between the two. The partial correlation coefficients were then averaged among the participants in each group to generate a partial correlation matrix.

\section{Results}

The partial correlation matrix for the Normal group clearly demonstrated the existence of three separate syntax-related networks (Figure 1a). Indeed, all of the functional connectivity within those networks was positive (mean $Z$ value, 0.18), while "cross-talk" between these networks was absent for the Normal group (mean $Z$ value, 0.002). We defined a "network-boundary effect" as the significantly greater connectivity among the regions within individual networks compared with other connectivity. The mean $Z$ values within individual networks were significantly greater than those between any two of the networks (one-tailed $t$ test, $P<0.0001$ ), confirming the network-boundary effects.

According to the partial correlation matrix for the LPMC and F3 groups, almost all of the functional 
Table 2 Patient demographics

\begin{tabular}{|c|c|c|c|c|c|}
\hline Patient & Age & Laterality quotient & Tumor location & Tumor type & Tumor grade \\
\hline \multicolumn{6}{|l|}{ LPMC group } \\
\hline Patient 1 & 31 & 88 & L. F1/F2/SMA/LPMC & $\mathrm{AO}$ & III \\
\hline Patient 2 & 47 & 81 & L. F1/F2/SMA/LPMC/F3op & $\mathrm{AOA}$ & III \\
\hline Patient 3 & 36 & 88 & L. F1/F2/SMA/LPMC/F3op & $\mathrm{AA}$ & III \\
\hline Patient 4 & 49 & 100 & L. F1/F2/LPMC & $\mathrm{AO}$ & III \\
\hline Patient 5 & 34 & 100 & L. F1/F2/LPMC/F3op & OD & $\|$ \\
\hline Patient 6 & 29 & 81 & L. F1/F2/LPMC/F3op & $\mathrm{AO}$ & III \\
\hline Patient 7 & 27 & 45 & L. F2/LPMC/F3op & DA & $\|$ \\
\hline Mean \pm SD & $36 \pm 8.6$ & $83 \pm 19$ & & & \\
\hline \multicolumn{6}{|l|}{ F3 group } \\
\hline Patient 8 & 32 & 54 & L. F1/F2/F3op/F3O/insula & $\mathrm{AOA}$ & III \\
\hline Patient 9 & 20 & 100 & L. F1/F2/F3op/F3O/insula & $\mathrm{AO}$ & III \\
\hline Patient 10 & 31 & 100 & L. F1/F2/F3op/F3t/insula/striatum & $\mathrm{AOA}$ & III \\
\hline Patient 11 & 32 & 100 & L. F1/F2/F3op/F3O/insula/striatum & DA & $\|$ \\
\hline Patient 12 & 42 & 90 & L. F2/F3op/F3O/insula/striatum & $\mathrm{OA}$ & $\|$ \\
\hline Patient 13 & 29 & 87 & L. F2/F3op/F3O/insula/striatum & OD & $\|$ \\
\hline Patient 14 & 47 & 73 & L. F3op/F3t/insula/striatum & DA & $\|$ \\
\hline Mean \pm SD & $33 \pm 8.8$ & $86 \pm 17$ & & & \\
\hline \multicolumn{6}{|l|}{ Other group } \\
\hline Patient 15 & 62 & 89 & L. F1/F2/SMA & $\mathrm{AO}$ & III \\
\hline Patient 16 & 24 & 68 & L. F1/F2/SMA/striatum & $\mathrm{OA}$ & $\|$ \\
\hline Patient 17 & 38 & 100 & L. F1/F2/SMA/striatum & $\mathrm{AOA}$ & III \\
\hline Patient 18 & 21 & 100 & L. F1/F2/F3t/striatum & DA & $\|$ \\
\hline Patient 19 & 29 & 100 & L. F1/F2/F3O/striatum & $\mathrm{AO}$ & III \\
\hline Patient 20 & 25 & 100 & L. F1/F2/F3t & DA & $\|$ \\
\hline Patient 21 & 36 & 100 & L. F2/F3O & OA & $\|$ \\
\hline Mean \pm SD & $34 \pm 14$ & $83 \pm 12$ & & & \\
\hline
\end{tabular}

The laterality quotient of handedness was determined by the Edinburgh handedness inventory (Oldfield 1971). The tumor type and grade were postoperatively and pathologically diagnosed by the World Health Organization Classification of Tumors of the Nervous System (2000).

$A A$ anaplastic astrocytoma (grade III), $A O$ anaplastic oligodendroglioma (grade III), $A O A$ anaplastic oligoastrocytoma (grade III), DA diffuse astrocytoma (grade II), $F 1$ superior frontal gyrus, $F 2$ middle frontal gyrus, $F 3 O$ orbital part of the inferior frontal gyrus, F3op opercular part of the inferior frontal gyrus, $F 3 t$ triangular part of the inferior frontal gyrus, L. left, LPMC lateral premotor cortex, OA oligoastrocytoma (grade II), OD oligodendroglioma (grade II), SD standard deviation, SMA supplementary motor area.

connectivity became abnormal, including that of the networks outside lesions (Figure 1b, c). Moreover, the network-boundary effects completely disappeared for both patient groups $(P>0.3)$. Based on the comparison of mean $Z$ values between each patient group and Normal group, both patient groups showed significantly decreased connectivity within individual networks (Dunnett test, $P<0.0001$ ), together with significantly increased connectivity between any two of the networks $(P<0.0003)$. Given such a leveling effect, the $Z$ values averaged among all region pairs were 0.045 and 0.052 for the LPMC and F3 groups, respectively; we regarded the higher $Z$ value of 0.052 as a typical value for abnormal connectivity.

According to the partial correlation matrix for the Other group (Figure 1d), some normal functional connectivity was preserved in the following region pairs (the first two regions listed for each network; see Table 1): the left F3op/F3t and left IPS ( $Z=0.22)$, the left LPMC and left AG $(Z=0.28)$, and the triangular and orbital parts of the left inferior frontal gyri (F3t and F3O) $(Z=0.25)$. Within individual networks, only these $Z$ values were significantly above the abnormal connectivity at $Z=0.052$ (one-tailed one-sample $t$ test among the Other group, $P<0.03)$. While the network-boundary effects were significant for the Other group $(P=0.026)$, this group showed significantly decreased connectivity within individual networks compared to the Normal group (Dunnett test, $P<0.0001$ ), together with significantly increased connectivity between any two of the networks $(P<0.0001)$. In addition, this group showed significantly increased connectivity between Networks III and I compared to the other network pairs (Tukey-Kramer test, $P<0.05)$. 


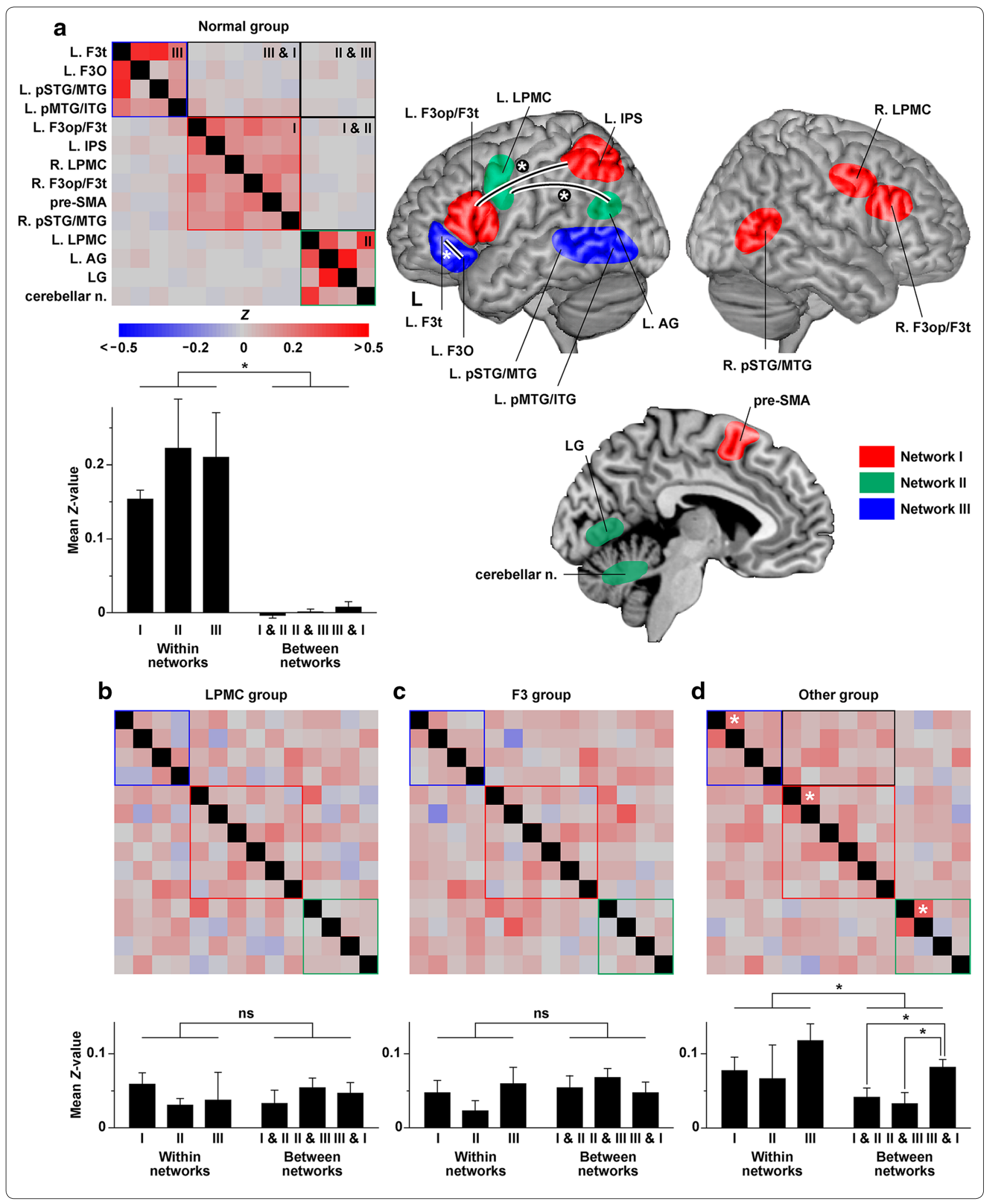




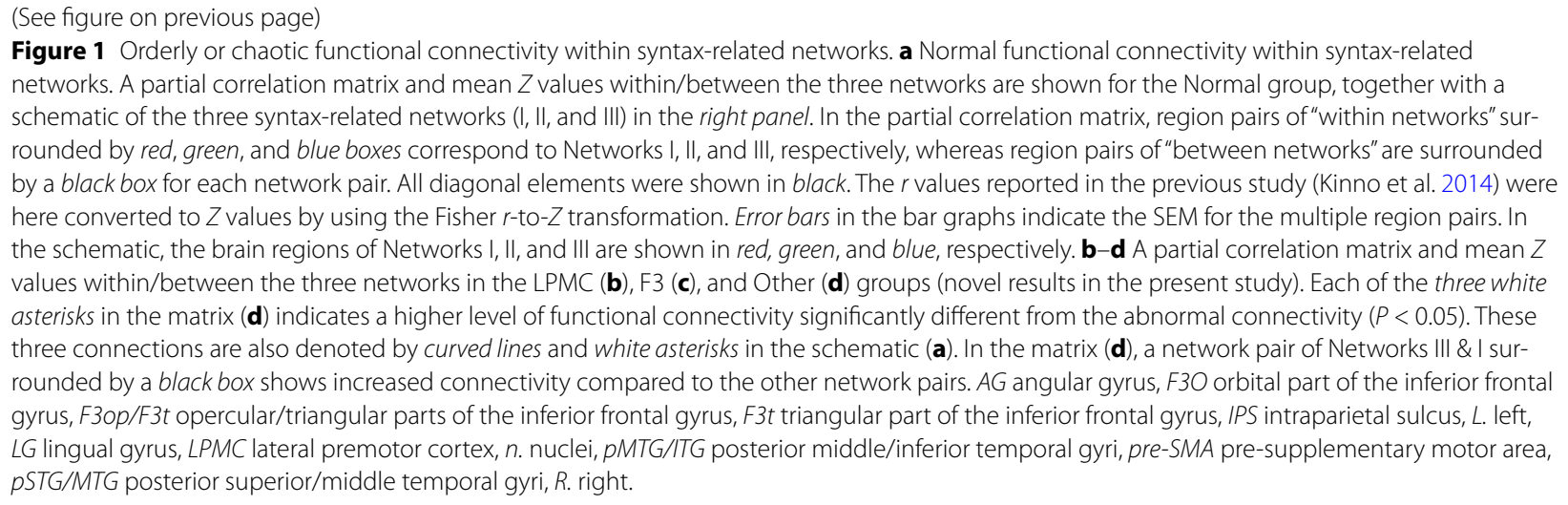

\section{Discussion}

We showed that the network-boundary effects were significant for both the Normal and Other groups (Figure 1a, d), while almost all of the functional connectivity exhibited chaotic changes in the LPMC and F3 groups (Figure 1b, c). It was notable that some functional connectivity was preserved in an orderly manner in the Other group, who showed normal task performances and activation patterns. More specifically, the Other group showed normal functional connectivity between the left F3op/F3t and left IPS (Network I), between the left LPMC and left AG (Network II), and between the left F3t and left F3O (Network III). Our results indicate that these pathways are most crucial among the syntaxrelated networks.

The crucial pathways within Networks I and II (Figure 1a), which were preserved in the Other group, actually pass through a dorsal pathway (i.e., the arcuate fasciculus and superior longitudinal fasciculus) in the left hemisphere. Some previous fMRI and diffusion MRI studies have suggested the importance of the dorsal pathway for syntactic processing (Friederici et al. 2006; Saur et al. 2008; Ohta et al. 2013). As regards the ventral pathway connecting the left F3t and left F3O, a recent study with fMRI and diffusion MRI has suggested that these regions make a triangulo-orbitaris system connected by intermingled U-shaped fibers (Lemaire et al. 2013). The integrity of both the dorsal and ventral pathways would thus be related to normal performances. The increased connectivity between Network III and I in the Other group may reflect rescuing interactions among these syntax-related networks to achieve normal syntactic processing.

We observed the leveling effect in all three of the patient groups (Figure 1b-d). We assume that the loss of functional connectivity observed in the present study reflects the globally-i.e., without any specific correlates of cognitive processes-increased excitability due to the upregulation of intrinsically bursting neurons within the peritumoral zone (de Groot et al. 2012). In spite of such abnormal excitability in the entire brain, it is surprising to note that both the LPMC and F3 groups showed distinct overactivity and/or underactivity among the regions within each of Networks I-III (Kinno et al. 2014). Moreover, these patient groups showed the different types of agrammatic comprehension, as well as differential activation patterns, although their abnormal functional connectivity was essentially similar. Furthermore, the Other group showed normal performances and activation patterns, even if functional connectivity was partially abnormal. Therefore, the global loss of functional connectivity may not be the primary cause of the abnormal performances and activation patterns; rather the loss of connectivity must be the complication of the glioma. The comparison of data between normal controls and patients would thus provide clinically valuable information, such as preserved functional connectivity and relevant functions. We believe that diagnoses utilizing such information would be effective to medical treatment including rehabilitation for intractable cognitive or behavioral deficits.

\section{Additional file}

Additional file 1. Supplementary Methods.

\section{Authors' contributions}

RK, YM, and TM collected the data. RK and SO analyzed the data. RK and KLS wrote the manuscript. All authors read and approved the final manuscript.

\section{Author details}

${ }^{1}$ Department of Basic Science, Graduate School of Arts and Sciences, The University of Tokyo, 3-8-1 Komaba, Meguro-ku, Tokyo 153-8902, Japan.

2 Division of Neurology, Department of Internal Medicine, Showa University Northern Yokohama Hospital, 35-1 Chigasaki-chuo, Tsuzuki-ku, Yokohama, Kanagawa 224-8503, Japan. ${ }^{3}$ CREST, Japan Science and Technology Agency, 7 Gobancho, Chiyoda-ku, Tokyo 102-0076, Japan. ${ }^{4}$ Department of Neurosurgery, Tokyo Women's Medical University, 8-1 Kawada-cho, Shinjuku-ku, Tokyo 162-8666, Japan. 


\section{Acknowledgements}

We thank N. Komoro for technical assistance and $\mathrm{H}$. Matsuda for administrative assistance. This work was supported by JST, CREST, by a Grant-in-Aid for Young Scientists (B) (No. 26870582) from MEXT, and by a grant from the Showa University School of Medicine Alumni Association.

\section{Compliance with ethical guidelines}

\section{Competing interests}

The authors declare that they have no competing interests.

\section{Consent for publication}

Written informed consent was obtained from each participant after the nature and possible consequences of the studies were explained.

\section{Ethical approval}

Approval for the experiments was obtained from the institutional review board of the University of Tokyo, Komaba, as well as that of the Tokyo Women's Medical University.

Received: 25 February 2015 Accepted: 17 June 2015

Published online: 04 July 2015

\section{References}

Ashburner J, Friston KJ (2005) Unified segmentation. Neuroimage 26(3):839-851

Bartolomei F, Bosma I, Klein M, Baayen JC, Reijneveld JC, Postma TJ et al (2006) How do brain tumors alter functional connectivity? A magnetoencephalography study. Ann Neurol 59(1):128-138
Briganti C, Sestieri C, Mattei PA, Esposito R, Galzio RJ, Tartaro A et al (2012) Reorganization of functional connectivity of the language network in patients with brain gliomas. Am J Neuroradiol 33(10):1983-1990

de Groot M, Reijneveld JC, Aronica E, Heimans JJ (2012) Epilepsy in patients with a brain tumour: Focal epilepsy requires focused treatment. Brain 135(4):1002-1016

Friederici AD, Bahlmann J, Heim S, Schubotz Rl, Anwander A (2006) The brain differentiates human and non-human grammars: Functional localization and structural connectivity. Proc Natl Acad Sci USA 103(7):2458-2463

Kinno R, Muragaki Y, Hori T, Maruyama T, Kawamura M, Sakai KL (2009) Agrammatic comprehension caused by a glioma in the left frontal cortex. Brain Lang 110(2):71-80

Kinno R, Ohta S, Muragaki Y, Maruyama T, Sakai KL (2014) Differential reorganization of three syntax-related networks induced by a left frontal glioma. Brain 137(4):1193-1212

Lemaire J-J, Golby A, Wells WM III, Pujol S, Tie Y, Rigolo L et al (2013) Extended Broca's area in the functional connectome of language in adults: Combined cortical and subcortical single-subject analysis using fMRI and DT tractography. Brain Topogr 26(3):428-441

Ohta S, Fukui N, Sakai KL (2013) Syntactic computation in the human brain: The Degree of Merger as a key factor. PLOS One 8(2):e56230

Oldfield RC (1971) The assessment and analysis of handedness: The Edinburgh inventory. Neuropsychologia 9(1):97-113

Saur D, Kreher BW, Schnell S, Kümmerer D, Kellmeyer P, Vry M-S et al (2008) Ventral and dorsal pathways for language. Proc Natl Acad Sci USA 105(46):18035-18040

Smith SM (2012) The future of FMRI connectivity. Neuroimage 62(2):1257-1266

\section{Submit your manuscript to a SpringerOpen ${ }^{\odot}$ journal and benefit from:}

- Convenient online submission

- Rigorous peer review

- Immediate publication on acceptance

- Open access: articles freely available online

- High visibility within the field

- Retaining the copyright to your article

Submit your next manuscript at $>$ springeropen.com 